\title{
Default Mode of Brain Activity Demonstrated by Positron Emission Tomography Imaging in Awake Monkeys: Higher Rest-Related than Working Memory-Related Activity in Medial Cortical Areas
}

\author{
Takashi Kojima, ${ }^{1}$ Hirotaka Onoe, ${ }^{1,2}$ Kazuo Hikosaka, ${ }^{1,3}$ Ken-ichiro Tsutsui, ${ }^{4}$ Hideo Tsukada, ${ }^{5}$ and Masataka Watanabe ${ }^{1}$ \\ ${ }^{1}$ Department of Psychology, Tokyo Metropolitan Institute for Neuroscience, Fuchu, Tokyo, 183-8526, Japan, ${ }^{2}$ Functional Probe Research Laboratory, \\ RIKEN Center for Molecular Imaging Research Science, Chuo-ku, Kobe, 650-0047, Japan, ${ }^{3}$ Department of Sensory Science, Faculty of Health Science and \\ Technology, Kawasaki University of Medical Welfare, Kurashiki, 701-0193, Japan, ${ }^{4}$ Division of Systems Neuroscience, Tohoku University Graduate School \\ of Life Sciences, Aoba-ku, Sendai, 980-8577, Japan, and ${ }^{5}$ Central Research Laboratory, Hamamatsu Photonics K.K., Hamakita-ku, Hamamatsu, 434-8601, \\ Japan
}

Human neuroimaging studies have demonstrated the presence of a "default system" in the brain, which shows a "default mode of brain activity," i.e., greater activity during the resting state than during an attention-demanding cognitive task. The default system mainly involves the medial prefrontal and medial parietal areas, including the anterior and posterior cingulate cortex. It has been proposed that this default activity is concerned with internal thought processes. Recently, it has been indicated that chimpanzees show high metabolic levels in these medial brain areas during rest. Correlated low-frequency spontaneous activity as measured by functional magnetic resonance imaging was observed between the medial parietal and medial prefrontal areas in the anesthetized monkey. However, there have been few attempts to demonstrate a default system that shows task-induced deactivation in nonhuman primates. We conducted a positron emission tomography study with $\left[{ }^{15} \mathrm{O}\right] \mathrm{H}_{2} \mathrm{O}$ to demonstrate a default mode of brain activity in the awake monkey sitting on a primate chair. Macaque monkeys showed higher level of regional blood flow in these medial brain areas as well as lateral and orbital prefrontal areas during rest compared with that under a working memory task, suggesting the existence of internal thought processes in the monkey. However, during rest in the monkey, the highest level of blood flow relative to that in other brain regions was observed not in the default system but in the dorsal striatum, suggesting that regions with the highest cerebral blood flow during rest may differ depending on the resting condition and/or species.

\section{Introduction}

Human neuroimaging studies have revealed the presence of a "default system" in the brain, which is more active during rest than during an attention-demanding cognitive task ("default mode of brain activity") (Raichle et al., 2001). A default activity is observed in the medial and lateral parietal cortex, anterior and posterior cingulate cortex, and medial prefrontal cortex (MPFC) (Shulman et al., 1997; Gusnard et al., 2001; Mazoyer et al., 2001; Raichle et al., 2001). During rest, regions within the default system show higher regional cerebral blood flow (rCBF) (Raichle et al., 2001) and higher resting glucose metabolism (Minoshima et

Received April 10, 2009; revised Sept. 4, 2009; accepted Sept. 21, 2009

This study was supported by a Grant-in-Aid for Scientific Research on Priority Areas (Integrative Brain Research) from the Ministry of Education, Culture, Sports, Science, and Technology of Japan (Grant 17022050) and by a Grant-in-Aid for Target-Orientated Research and Development in Brain Science from the Japan Science and Technology Corporation (Grant 13073-2125). We thank T. Kakiuchi and D. Fukumoto of Hamamatsu Photonics for technical assistance while conducting the PET experiments and K. Christoff for comments on the early version of this manuscript.

Correspondence should be addressed to Masataka Watanabe, Department of Psychology, Tokyo Metropolitan Institute for Neuroscience, 2-6 Musashidai, Fuchu, Tokyo, 183-8526, Japan. E-mail: watanabe-ms@igakuken.or.jp. D01:10.1523/JNEUROSCI.1786-09.2009

Copyright $\odot 2009$ Society for Neuroscience $\quad$ 0270-6474/09/2914463-09\$15.00/0 al., 1997) relative to those of other brain regions. Correlated spontaneous activity as measured by functional magnetic resonance imaging (fMRI) is observed across areas within the default system (Fox et al., 2005; Fransson, 2005). The default activity is thought to be concerned with internal thought processes (Christoff et al., 2004), such as the recall of autobiographical episodic memories (Mazoyer et al., 2001), self-referential processing (Kelley et al., 2002), conceptual processing (Binder et al., 1999), spontaneous semantic processing (McKiernan et al., 2003), mind-wandering (Mason et al., 2007), and monitoring of the external environment, body image, and emotional state (Gusnard et al., 2001). Default activity is abnormal in patients with autism (Kennedy et al., 2006), Alzheimer's disease (Lustig et al., 2003), depression (Greicius et al., 2007), schizophrenia (Liang et al., 2006), and attention deficit hyperactivity disorder (Tian et al., 2006). This default activity is not well developed in children (Fair et al., 2008).

To clarify the functional significance of the default activity, it is important to examine whether a similar default system exists in the nonhuman primate that does not have linguistic ability. A recent positron emission tomography (PET) study with 2-deoxy-2 $\left[{ }^{18} \mathrm{~F}\right]$ fluoro-D-glucose ([ $\left.\left.{ }^{18} \mathrm{~F}\right] \mathrm{FDG}-\mathrm{PET}\right)$ in the chimpanzee dem- 
onstrated that the highest level of metabolic activity during rest occurred in the medial and lateral prefrontal areas and medial and lateral parietal areas (Rilling et al., 2007). In the anesthetized monkey, low-frequency, spontaneous activity measured by fMRI showed a correlation between the medial parietal and medial prefrontal areas (Vincent et al., 2007). A recent study demonstrated task-induced suppression of neuronal activity in the monkey posterior cingulate cortex (Hayden et al., 2009). However, there has not been any PET or fMRI study in the nonhuman primate indicating the presence of a default system showing taskinduced deactivation. It is not clear whether regions showing the highest level of metabolic activity during rest or regions with correlated spontaneous activity within the medial brain areas in nonhuman primates correspond to regions showing taskinduced deactivation. To directly locate the default brain activity in the monkey, we conducted a PET study on unanesthetized monkeys with $\left[{ }^{15} \mathrm{O}\right] \mathrm{H}_{2} \mathrm{O}$ that can measure the changes in $\mathrm{rCBF}$. We compared the resting brain activity with the brain activity during the working memory (WM) task. We also examined regions that showed the highest level of blood flow during rest and compared these regions with those regions showing task-induced deactivation.

\section{Materials and Methods}

Three Japanese macaque monkeys (Macacca fuscata, 5.5-7.0 kg) were used in this study. Monkeys A and B were trained with juice reward on spatial and nonspatial delayed response (DR) tasks (spatial-DR and nonspatial-DR), as well as on spatial and nonspatial control (CON) tasks without delays (spatial-CON and nonspatial-CON) (supplemental Fig. 1, available at www.jneurosci.org as supplemental material). Monkey C was trained and tested with either water or juice reward only on the two spatial tasks. The monkey was seated in a primate chair facing a cathode ray tube (CRT) display (15 inch; NEC). In front of the monkey, there were three keys arranged horizontally (right, center, and left) at the animal's waist level. In the spatial-DR task, the monkey first pressed the center key for $1 \mathrm{~s}$ [intertrial interval (ITI)] with its right hand, and then a positional cue consisting of a blue rectangle was presented for $0.5 \mathrm{~s}$ on either the right or left side of the display. Then, there was a delay period of $4 \mathrm{~s}$. When the delay period was over, white lights appeared on both the right and left sides of the display as a "go" signal. When the monkey pressed the key on the side where the positional cue had been presented, a drop of orange juice $(0.3 \mathrm{ml})$ (in the case of monkey $\mathrm{C}$, either juice or water) was delivered as a reward. In the spatial-CON task, the duration of the ITI was $5 \mathrm{~s}$, and there was no delay period.

In the nonspatial-DR and nonspatial-CON tasks, instead of the positional cue, a two-dimensional cue was presented at the center of the display for $0.5 \mathrm{~s}$. In the nonspatial-DR task, after the cue presentation, there was a delay period of $4 \mathrm{~s}$, after which white lights were presented on both the right and left sides of the display as a go signal. In the nonspatialCON task, the ITI was $5 \mathrm{~s}$, and there was no delay period. In both tasks, after the go signal, the monkey had to respond by pressing the right key when the cue was a circle and the left key when the cue was stripes. The task was controlled by two personal computers (PC9801FA; NEC). The monkeys were fully trained to attain nearly $100 \%$ accuracy in daily training. During the experiments, which were conducted on weekdays, the monkeys obtained all their fluid during the tasks but were given access to water ad libitum for $>24 \mathrm{~h}$ during each weekend. Monkey pellets were available at all times in the home cage.

After training, monkeys underwent PET scanning in a non-illuminated experimental room during all task conditions. We previously reported behavioral data as well as brain activities in relation to the performance of these tasks (Kojima et al., 2007). Monkeys were also scanned during rest (REST), while they sat quietly on the monkey chair without task performance. There were no visual stimuli presented, and the CRT display was only dimly illuminated during REST.

All experiments were conducted in accordance with the National Institutes of Health guidelines for animal experiments as well as the insti- tutional guidelines of the Central Research Laboratory of Hamamatsu Photonics and were approved by the ethics committee of the Tokyo Metropolitan Institute for Neuroscience.

The monkey was allowed to begin the task $30 \mathrm{~s}$ before the start of each PET scan and continued throughout scanning. During the PET scan, monkeys A and B performed one of four tasks (spatial-DR, spatial-CON, nonspatial-DR, and nonspatial-CON) or sat quietly (REST). Monkey C performed one of two tasks (spatial-DR and spatial-CON) with either orange juice or water as a reward $(2 \times 2$ factor) or sat quietly (REST). Movements of the left hand and the right and left legs of the monkey were lightly restrained by loosely binding them to the chair with cotton cloth. In a single daily session, the four tasks (in monkeys A and B) or two tasks with one of two types of reward (in monkey $\mathrm{C}$ ) and the REST condition were performed at $\sim 15 \mathrm{~min}$ intervals, and $20-25$ successive PET images were obtained. On each day, a predefined semi-random sequence determined which type of task (in the case of monkey C, which task and reward) and REST condition the monkey would participate in during each PET scan to eliminate any effect of the order of the four types of task and the REST condition. Thus, each monkey underwent PET scanning for a total of $6-7 \mathrm{~d}$ with four to five scans being obtained under each condition on each day, resulting in a total of 29-31 successive PET images obtained under each condition.

Surgery and PET study. After the training was completed, T1-weighted MRI images of each monkey were obtained with either a 0.5 tesla unit [MRT-50A/II (Toshiba); resolution, $0.586 \times 0.586 \times 3 \mathrm{~mm} /$ pixel; monkeys $\mathrm{A}$ and $\mathrm{B}$ ] or a 0.3 tesla unit [AILIS2-1 (Hitachi); resolution, $0.703 \times 0.703 \times 3 \mathrm{~mm} /$ pixel; monkey C). After scanning, an acrylic head-restraining device was attached to the monkey under pentobarbital anesthesia. After partially exposing the skull, stainless steel screws were used to firmly attach the head-restraining device to the skull. The monkey was given antibiotics every day for a week after the surgery.

Before the PET activation studies with $\left[{ }^{15} \mathrm{O}\right] \mathrm{H}_{2} \mathrm{O}$, we obtained an $\left[{ }^{18} \mathrm{~F}\right]$ FDG-PET image for each monkey by injecting a bolus of $\left[{ }^{18} \mathrm{~F}\right] \mathrm{FDG}$ ( $40 \mathrm{MBq} / \mathrm{kg}$ in $5 \mathrm{ml}$ of saline). Before the PET scanning with $\left[{ }^{15} \mathrm{O}\right] \mathrm{H}_{2} \mathrm{O}$ each day, the monkey was transferred to the monkey chair without anesthesia, and a venous cannula was placed in the sural vein. PET scans were performed with an animal PET scanner (SHR-7700; Hamamatsu Photonics) while the monkey's head was rigidly fixed to the frame of the monkey chair using the acrylic head-restraining device. Each monkey's head was placed in precisely the same position throughout the study session including the $\left[{ }^{18} \mathrm{~F}\right] \mathrm{FDG}$ imaging.

At the start of the daily PET experiment, a 30 min transmission scan with a rotating ${ }^{68} \mathrm{Ge}-{ }^{68} \mathrm{Ga}$ pin source was obtained to evaluate the relative attenuation factor for image reconstruction. At each PET scan, a bolus of $\left[{ }^{15} \mathrm{O}\right] \mathrm{H}_{2} \mathrm{O}(\sim 1.2 \mathrm{GBq}$ in $1.5 \mathrm{ml}$ of saline), followed by $1.0 \mathrm{ml}$ of saline, was delivered within $15 \mathrm{~s}$ through the venous cannula using an automatic injector. Each PET scan was initiated automatically when radioactivity in the brain reached over 30 kilo counts per second. Data were collected for 120 s divided into six 20 -s epochs. Thirty-one slices with a center-to-center distance of $3.6 \mathrm{~mm}$ were collected simultaneously, covering the whole brain of the monkey. The axial and transaxial resolution of the PET scanner was $2.6 \mathrm{~mm}$ at full-width half-maximum in the enhanced two-dimensional mode. Tilting the PET camera gantry (parallel to the orbito-meatal line) allowed the monkey to sit in an upright position, making it possible for the monkey to view the CRT display. Image reconstruction was performed on projection data, after which images were corrected for attenuation using a transmission scan with a $4.5 \mathrm{~mm}$ Hanning filter (SHR Control II; Hamamatsu Photonics). Reconstructed brain images from each study were scalped and smoothed using a $3.0 \mathrm{~mm}$ full-width half-maximum isotropic kernel. To determine the anatomical localization of activated foci, we performed a combination of accurate stereotaxic measurements of the MRI and PET images. Using a threedimensional alignment program (3D BrainStation; Loats Associates), we coregistered the $\operatorname{SPM}\{Z\}$ PET image with the matching MRI image along the cortical borderline determined by the $\left[{ }^{18} \mathrm{~F}\right] \mathrm{FDG}$-PET image.

Statistical analyses. Data summated over the first $60 \mathrm{~s}$ were used for additional statistical analyses according to the method described by Onoe et al. (2001). The global activity for each scan was corrected using grand mean scaling, using an analysis of covariance for global normalization. 
Monkey A

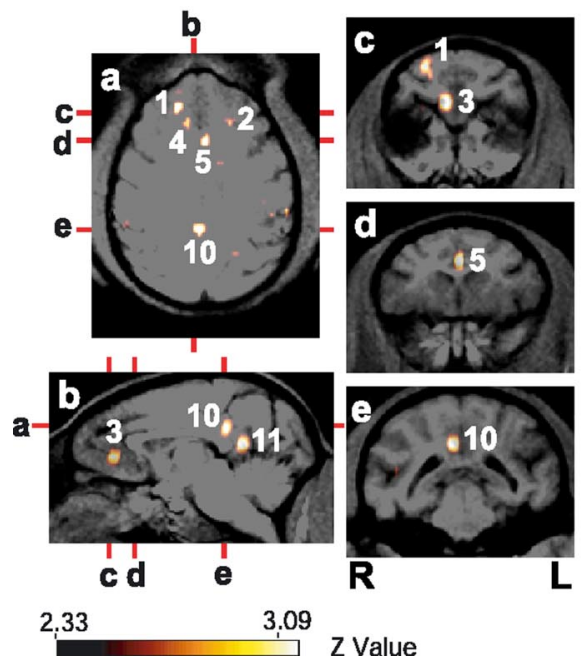

Monkey B
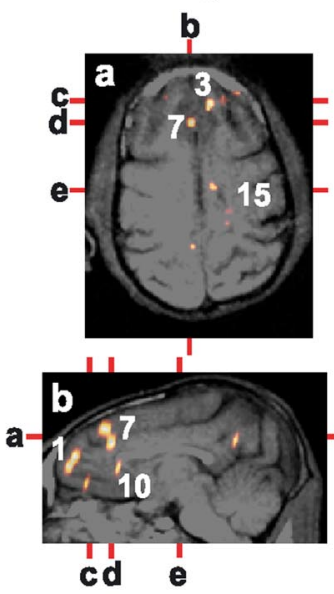
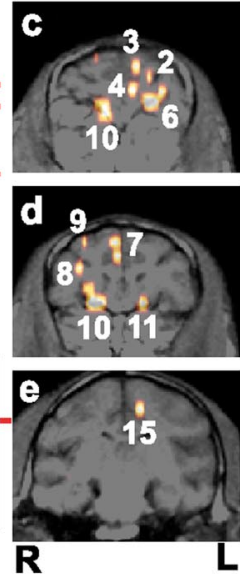

Monkey C
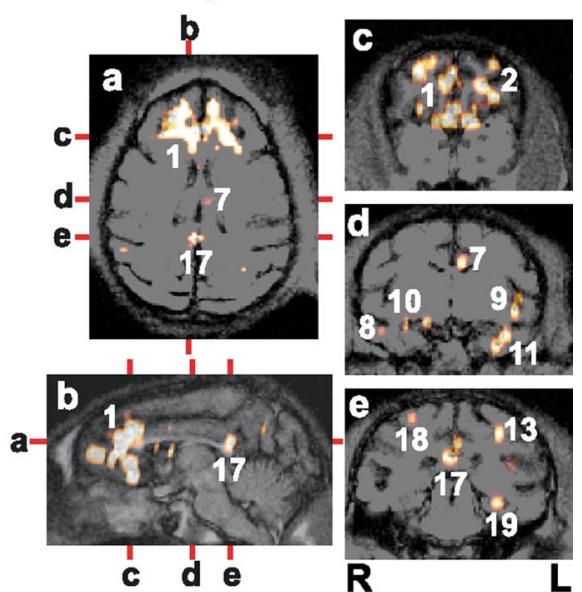

Figure 1. Regions with higher activity during REST than during the spatial WM task. Subtraction images are shown separately for each monkey. Top left ( $\boldsymbol{a}$ ), bottom left ( $\boldsymbol{b})$, and right three ( $\boldsymbol{c}-\boldsymbol{e}$ ) images indicate transverse, sagittal, and coronal brain sections of each monkey, respectively. Vertical line $\boldsymbol{b}$ in the top left indicates the left (L)-right (R) line corresponding to the sagittal section pictured in the bottom left $(\boldsymbol{b})$. Horizontal line a in $\boldsymbol{b}$ indicates the top-bottom line corresponding to the transverse section pictured in $\boldsymbol{a}$. Lines $\boldsymbol{c}-\boldsymbol{e}$ in $\boldsymbol{a}$ and $\boldsymbol{b}$ indicate the anterior-posterior line corresponding to the coronal sections pictured in the right panels $(\boldsymbol{c}-\boldsymbol{e})$. The numbers in each panel represent the locations of higher activity during REST and correspond to the numbers in supplemental Table $1 A$ (available at www.jneurosci.org as supplemental material).

After normalizing the injected radioactivity, statistical analysis of the reconstructed PET images (pixel size, $1.2 \times 1.2 \mathrm{~mm}$ ) was performed using Statistical Parametric Mapping (SPM99) software (Wellcome Department of Cognitive Neurology, University College London, London, UK) implemented in MATLAB (MathWorks). The difference in the relative $\mathrm{rCBF}$ between each of the four task conditions and the resting state, such as between the spatial-DR and REST conditions, was statistically tested in each voxel for data from each monkey individually with 24 repetitions per condition. Based on the transverse, coronal, and sagittal images of the subtraction images of each monkey, we noted the brain regions that showed significantly different activities (statistical threshold set at $Z=3.09$, uncorrected for multiple comparisons, $p<0.001$ ) between each of the four tasks (in monkey $\mathrm{C}$, the two spatial tasks with the juice reward) and the resting condition. Because of the limited spatial resolution, any region consisting of $<4$ clustered voxels whose signals were above the threshold of $Z=3.09$ was not considered a significant region. In addition, we conducted conjunction analysis to show overlap of deactivations across tasks (spatial-DR, spatial-CON, nonspatial-DR, and nonspatial-CON) within each monkey as well as multi-subject analysis to obtain the average location of deactivations in the spatial and nonspatial tasks across individual monkeys. For the multi-subject analysis, brain shapes of individual monkeys were morphologically normalized to a pseudo-brain template of [18F]FDG-PET image, which was constructed by averaging images of three monkeys used in the present study. For the multi-subject analysis, the criterion for statistical significance was set at $p<0.05$ (corrected for multiple comparisons).

We identified the anatomical locations of the active foci by referring to the atlas of the rhesus monkey brain (Paxinos et al., 2000; Saleem and Logothetis, 2007). Comparison of activities for each region among the four different tasks and REST in each monkey was conducted by one-way ANOVA and post hoc analysis using Ryan's method. The criterion for statistical significance was set at $p<0.05$.

To determine whether there were significant differences among the four kinds of "rest-related activity" (higher activity during REST than during task performance), we compared the number of voxels in each region showing rest-related activity separately for monkey A and monkey B using a two-way (task $\times$ region) ANOVA. Similarly, to find out whether there were significant differences among the four kinds of taskrelated activation (higher activity during task performance than during REST), we compared the number of voxels in each region showing taskrelated activation for monkey A and monkey B using a two-way (task $\times$ region) ANOVA. For monkey C, which did not perform the nonspatial tasks, comparisons were performed only on the data obtained during the juice reward condition. To examine further whether there were significant differences in the number of voxels in each region with rest-related activity (task-induced deactivation) between two different task conditions, we conducted $\chi^{2}$ tests on rest-related activities comparing between the DR and CON tasks and between the spatial and nonspatial tasks for each monkey. To examine whether there were significant differences in the numbers of voxels in each region with task-related activation between two different task conditions, we conducted $\chi^{2}$ tests on task-related activities comparing between DR and CON tasks and between the spatial and nonspatial tasks for each monkey.

Examination of areas showing high levels of $r C B F$ during REST. We also examined areas showing the highest levels of $\mathrm{rCBF}$ during the resting period. By using the image-averaging utilities of SPM99, we obtained normalized average rCBF data for each voxel for 24 successive scans taken during the REST condition. Then, using the 3D BrainStation program, we coregistered the normalized average $\mathrm{rCBF}$ value of each voxel with the matching MRI image. By applying several threshold values (5, 10 , and $20 \%$ ) to the whole brain, we obtained images of the regions showing relatively high levels of rCBF during REST.

\section{Results}

To compare the brain activity of monkeys during REST with that during the spatial-DR task, subtraction images [(REST - spatial$\mathrm{DR})$ ] were made for each monkey. In all three monkeys (A, B, and $\mathrm{C})$, higher activities were observed during REST than during the spatial-DR task in the lateral PFC (LPFC), MPFC, anterior cingulate cortex (ACC), orbitofrontal cortex (OFC), and posterior cingulate cortex/precuneus area (PCC/precuneus) (Fig. 1) (supplemental Fig. 2 and Table $1 A$, available at www.jneurosci.org as supplemental material). Higher activities during REST were also observed in the auditory cortex and insula in monkey $\mathrm{A}$ and monkey $\mathrm{C}$ and in the striatum in monkey $\mathrm{B}$ and monkey $\mathrm{C}$.

Reverse subtraction of (spatial-DR - REST) identified the regions that were more active during the spatial-DR task (Fig. 2) (supplemental Table $1 B$, available at www.jneurosci.org as supplemental material). During the task, monkeys looked at the CRT display, moved their right hand, and licked the tube to obtain the 
Monkey A

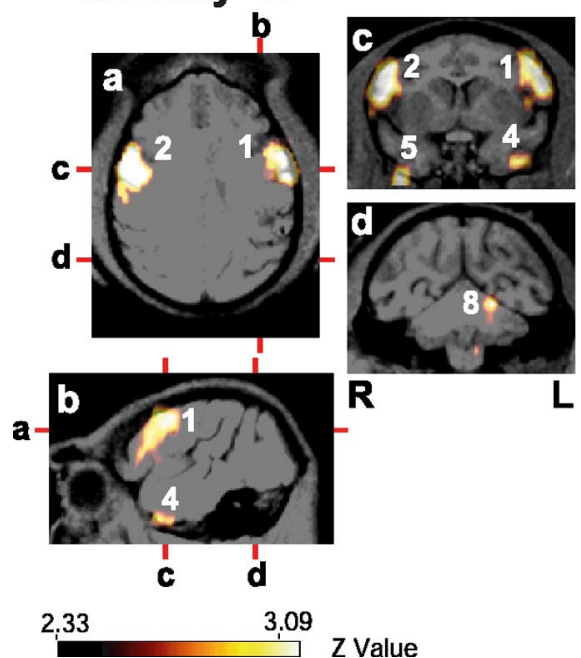

Monkey B

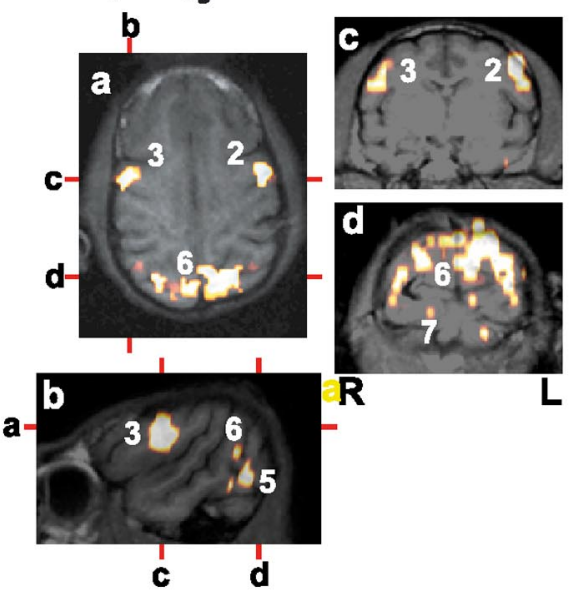

Monkey C

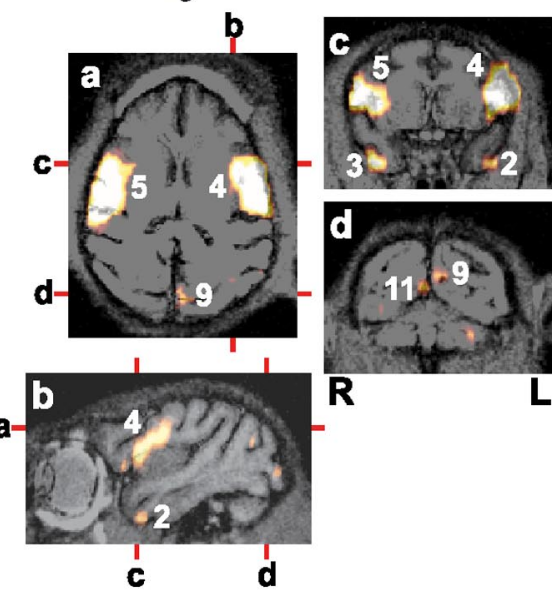

Figure 2. Regions with higher activity during the spatial-DR task than during REST. Subtraction images of (spatial-DR - REST) are illustrated for each monkey. Top left ( $\boldsymbol{a}$ ), bottom left ( $\boldsymbol{b}$ ) and right two $(\boldsymbol{c}, \boldsymbol{d})$ images indicate transverse, sagittal, and coronal sections of each monkey, respectively. Numbers in each panel represent the locations of higher activity during the spatial-DR task and correspond to the numbers in supplemental Table $1 B$ (available at www.jneurosci.org as supplemental material). Other conventions are the same as in Figure 1.

liquid reward. In all monkeys, higher activities were observed in the sensorimotor, ventral premotor, and visual areas (mainly in the visual association cortex TE in monkey A and in the primary and secondary visual cortices in monkey B and monkey C). Higher activities were also observed in the cerebellum in monkey $\mathrm{A}$ and monkey $\mathrm{C}$.

Monkeys were also trained in a spatialCON task. We obtained subtraction images of (REST - spatial-CON) and (spatial-CON - REST) for each monkey. Although there was no WM requirement in the control task, we found that there was no significant difference in subtraction image between (REST - spatialCON) and (REST - spatial-DR) or between (spatial-CON - REST) and (spatial-DR - REST) ( $\chi^{2}$ test, $\left.p>0.05\right)$; the regions showing higher activity during REST than during spatial-CON were almost identical to the regions that were more active during REST compared with the spatial-DR task, in terms of both their location and extent (supplemental Fig. 3A, available at www. jneurosci.org as supplemental material). Regions with higher activity during spatial-CON compared with REST were also almost the same as regions that were more active in the spatial-DR versus REST contrast (supplemental Fig. 3B, available at www.jneurosci. org as supplemental material).

Besides the spatial-DR and spatial-CON tasks, monkey A and monkey B performed nonspatial-DR as well as nonspatial-CON tasks during the PET study. For both monkeys, there was no significant difference in subtraction image between (REST nonspatial-DR) and (REST - spatial-DR) or between (nonspatial-DR - REST) and (spatial-DR - REST) $\left(\chi^{2}\right.$ test, $\left.p>0.05\right)$. Thus, regions with higher activity during REST than during the nonspatial-DR task were very similar in location and extent to the
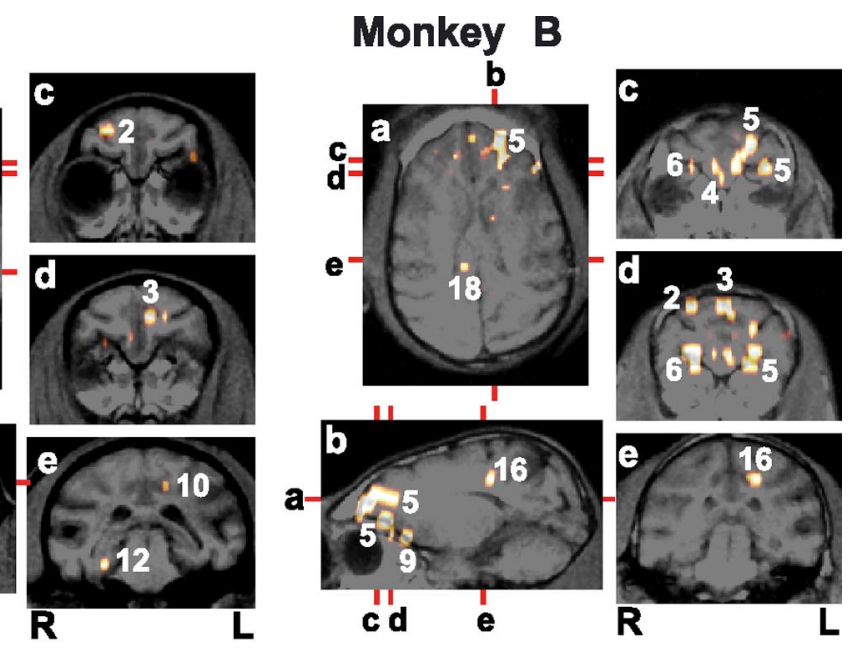

Figure 3. Regions with higher activity during REST than during the nonspatial WM task. Subtraction images of (REST nonspatial-DR) are shown separately for monkey $A$ and monkey B. Numbers in each panel represent the locations of higher activity during REST and correspond to the numbers in supplemental Table $2 A$ (available at www.jneurosci.org as supplemental material). Other conventions are the same as in Figure 1.

regions that showed higher activity during REST compared with the spatial-DR task (Fig. 3) (supplemental Table $2 A$, available at www.jneurosci.org as supplemental material). Also, regions with higher activity during the nonspatial-DR task versus REST were similar to the regions showing higher activity when the spatial-DR task was contrasted with REST (Fig. 4) (supplemental Table $2 B$, available at www.jneurosci.org as supplemental material). Similarly, subtraction and reverse subtraction images between REST and nonspatial-CON were similar to those subtraction images between REST and nonspatial-DR in terms of location and extent (supplemental Fig. 4, available at www.jneurosci.org as supplemental material).

In the conjunction analysis, we obtained subtraction images of $[($ REST - spatial-DR $)+($ REST - spatial-CON $)+($ REST nonspatial-DR $)+($ REST - nonspatial-CON)] for each monkey 


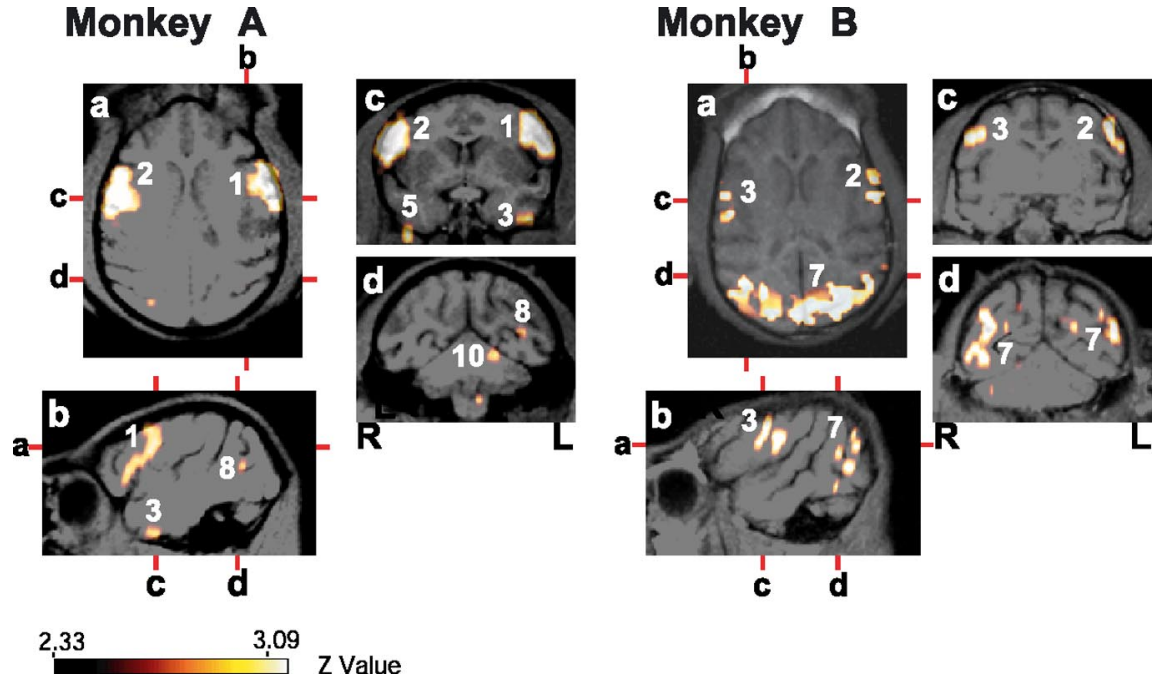

Figure 4. Regions with higher activity during the nonspatial-DR task than during REST. Subtraction images of (nonspatial$D R-$ REST) are illustrated for monkey $A$ and monkey $B$. Numbers in each panel represent locations of higher activity during the nonspatial-DR task and correspond to the numbers in supplemental Table $2 B$ (available at www.jneurosci.org as supplemental material). Other conventions are the same as in Figure 1.

(Fig. 5) (supplemental Table 3, available at www.jneurosci.org as supplemental material). As were found in the subtraction of [REST - (any kind of) task], activities during REST were higher in LPFC, MPFC, ACC, OFC, and PCC/precuneus for all three monkeys. As indicated in supplemental Table 3 (available at www. jneurosci.org as supplemental material), the lateral posterior parietal and visual association areas were also more active during REST in all monkeys.

In the multi-subject analysis, we obtained the average location of spatial task-induced deactivations by averaging data of three monkeys (A, B, and C) concerning [(REST - spatial-DR) + (REST - spatial-CON)] (Fig. 6A), as well as that of nonspatial task-induced deactivations by averaging data of two monkeys (A and B) concerning [(REST - nonspatial-DR) + (REST - nonspatial-CON)] (Fig. 6B). Average locations of spatial taskinduced deactivations across the three monkeys were quite similar to the regions that were more active during REST compared with the spatial-DR or spatial-CON task in each monkey; all the LPFC, OFC, ACC, MPFC, and PCC/precuneus areas showed taskinduced deactivations. Additional deactivations were also observed in the lateral posterior parietal area and insula. Average locations of nonspatial task-induced deactivations in two monkeys ( $\mathrm{A}$ and $\mathrm{B}$ ) were also similar to the regions that were more active during REST compared with the nonspatial-DR or nonspatial-CON task in each monkey, except that the PCC/precuneus area did not show significant deactivation. Additional deactivations were observed also in the lateral posterior parietal, visual association, and motor areas.

For all regions that showed higher activity during REST than during the spatial-DR task (supplemental Table $1 A$, available at www.jneurosci.org as supplemental material), we conducted a one-way ANOVA on the PET data and found that there were significant differences in activity among the five different (four tasks and REST) conditions for all monkeys $(p<0.01)$. However, subsequent post hoc comparisons using Ryan's methods on the activity in each region between the WM and control tasks for all monkeys and between spatial and nonspatial tasks (of either the WM or control condition) for monkey A and monkey B demonstrated that there were no regions in which a significant difference $(p<0.05)$ in activity between the two different task conditions was observed. Thus, in those regions showing spatial-DR task-induced deactivation, there was neither significant WM-related activity (higher activity in the delayed than in the control task) nor differential activity between spatial and nonspatial tasks (in monkeys A and B).

To determine whether the magnitude of the rest-related activity (i.e., of the taskinduced deactivation) differed depending on the task condition, we compared the total number of voxels showing taskinduced deactivation among the four task conditions for all three animals (supplemental Tables 1, 2, available at www. jneurosci.org as supplemental material). Two-way (monkey $\times$ task) ANOVA demonstrated a significant difference in the animal factor $(p<0.01)$ but not in the task factor or the task $X$ animal interaction $(p>0.05)$. Post hoc analyses indicated that there were significant differences in the total numbers of voxels showing taskinduced deactivation between any two monkeys $(p<0.01)$. However, there was no significant difference in the number of voxels between the four kinds of rest-related activity for all monkeys $(p>0.05)$.

We previously reported brain regions that showed spatial WM-related activity (higher activity during the spatial-DR task than during the spatial-CON task) outside the LPFC area (Kojima et al., 2007). We examined whether the spatial-DR taskinduced deactivation was present in regions showing spatial WM-related activity. Although some of the brain areas (ACC, insula, and PCC in monkey A; PCC and striatum in monkey B; and the MPFC in monkey C) in which spatial WM-related activity was observed were active during REST, the exact locations of WM-related activity did not overlap with those of rest-related activity (Fig. 1) (Kojima et al., 2007, their Fig. 2).

Our previous study reported nonspatial WM-related activity in several PFC areas (Kojima et al., 2007). We examined the restrelated activity (nonspatial-DR task-induced deactivation) in the $\mathrm{PFC}$ areas that showed nonspatial WM-related activity. In monkey $\mathrm{A}$ and monkey $\mathrm{B}$, we found that there was no overlap between the areas showing nonspatial WM-related activity and the areas with rest-related activity (Fig. 3) (Kojima et al., 2007, their Fig. 3).

To examine the regions that showed the highest levels of $\mathrm{rCBF}$ during REST in the present $\left[{ }^{15} \mathrm{O}\right] \mathrm{H}_{2} \mathrm{O}$-PET study, we first identified the top 5\% most active voxels in the whole brain (Fig. 7A) for each monkey. In all monkeys, the highest level of activity was observed in the dorsal striatum, but notable activity outside the striatum was observed only in one monkey (monkey A) in the somatosensory area, primary visual and visual association cortices, insula, and PCC. In contrast to the chimpanzee study on resting metabolism (Rilling et al., 2007), the medial PFC and medial parietal regions were not sufficiently active to meet $5 \%$ or even $10 \%$ threshold levels. When we used a more liberal threshold and highlighted the top 20\% most active voxels for each monkey, in addition to the striatum, all monkeys demonstrated higher-level $\mathrm{rCBF}$ in the medial brain areas that showed taskinduced deactivation (i.e., the ACC, PCC/precuneus, and MPFC) (Fig. $7 B$ ). Higher levels of rCBF were also observed in all monkeys in the insula, thalamus, cerebellum, and primary visual and visual association cortices. In monkeys A and B, we observed higher 
Monkey A
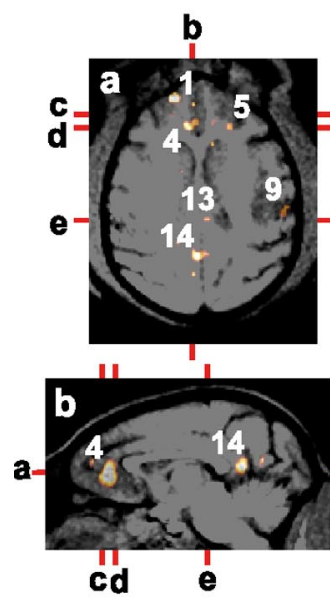

2.33
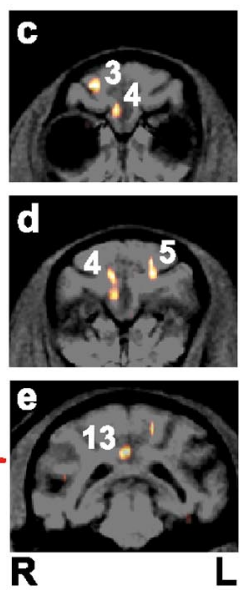
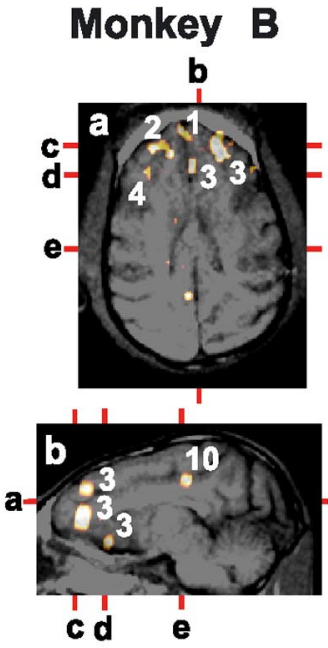
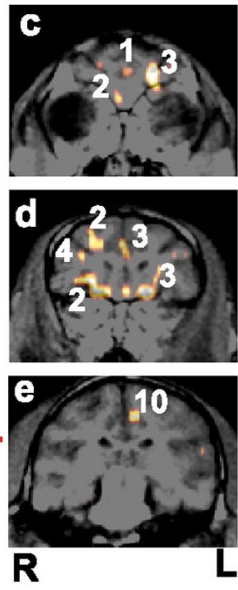
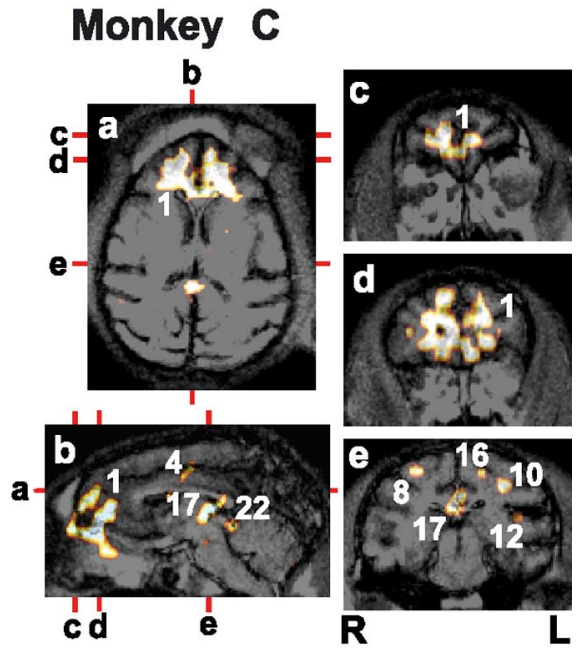

Figure 5. Regions with higher REST-related activity than average activity related to the performance of four different tasks. Subtraction images of $[($ REST - spatial-DR) + (REST - spatial-CON) + (REST - nonspatial-DR) + (REST - nonspatial-CON)] are shown separately for each monkey. Numbers in each panel represent the locations of higher activity during REST and correspond to the numbers in supplemental Table 3 (available at www.jneurosci.org as supplemental material). Other conventions are the same as in Figure 1.

levels of rCBF in the LPFC, premotor, and sensorimotor areas. There was no highlevel rCBF observed in the OFC in any of the monkeys.

\section{Discussion}

Human default activity has been observed predominantly in medial parts of the brain (the anterior medial prefrontal and posterior medial parietal areas) (Raichle et al., 2001). Similar to the human default system, all monkeys showed higher restrelated activity in the medial prefrontal and medial parietal areas. Functional roles of medial brain areas may differ between the monkey and human. However, in the monkey, the medial prefrontal area is involved in monitoring behavioral outcomes, especially in social contexts (Rushworth et al., 2007), and the medial parietal area is concerned with outcome evaluation and subsequent behavioral modification (Hayden et al., 2008). Thus, there are some functional similarities between the medial brain areas in humans (Gusnard et al., 2001) and those in monkeys. It has been proposed that activity in the human default system is related to internal thought processes (Christoff et al., 2004). Thus, the results of the present study demonstrating also default activity in the medial brain areas in the monkey suggest that there might be internal thought processes in the monkey. Of course, it is not known what the exact nature of the internal thought might be, given the lack of linguistic ability in monkeys. Future studies are needed to conduct PET or fMRI studies to examine what kind of cognitive operations are associated with increased activity in the monkey default system.

The lateral posterior parietal area, which is part of the human default system (Shulman et al., 1997), involves angular and supramarginal gyri that are concerned with linguistic processing

\section{A REST minus spatial-task B REST minus nonspatial-task}
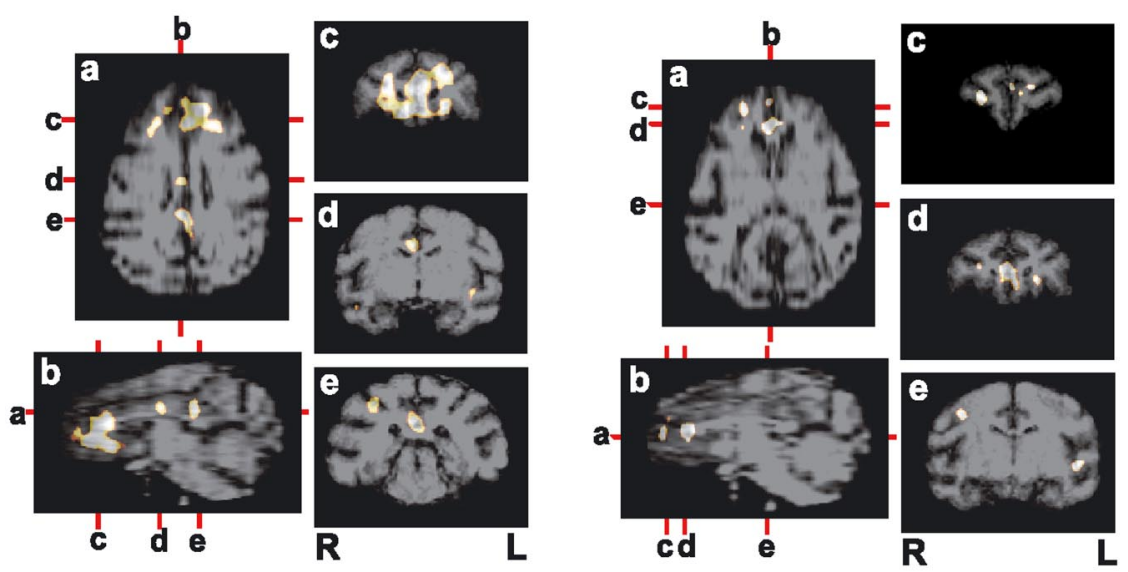

Figure 6. Average locations of deactivations in the spatial and nonspatial tasks across individual monkeys. $A$, Regions with higher REST-related activity than average activity related to the performance of spatial (both DR and CON) tasks across three monkeys (A, B, and C). $B$, Regions with higher REST-related activity than average activity related to the performance of nonspatial (both DR and CON) tasks across monkey A and monkey B. The deactivations are superimposed on a three-dimensional reconstruction of a template brain MRI that was produced from Japanese macaque monkeys used in the present study. Other conventions are the same as in Figure 1.

and may support internal thought. In the present study, taskinduced deactivation was observed in the lateral posterior parietal area only during limited kinds of tasks in monkeys A and C. However, when convergence images across the four different tasks were obtained (supplemental Table 3, available at www. jneurosci.org as supplemental material), clear task-induced deactivation was observed in this brain area in all monkeys. Thus, the lateral posterior parietal area also appears to constitute the default system in the monkey, but the roles of this area in the default system may not be as critical as those in humans.

It is notable that we observed consistently higher rest-related activity in the lateral and orbital PFC besides the medial brain areas in the monkey. The monkey LPFC is thought to be con- 
A

\section{Regions with top $5 \%$ activity}

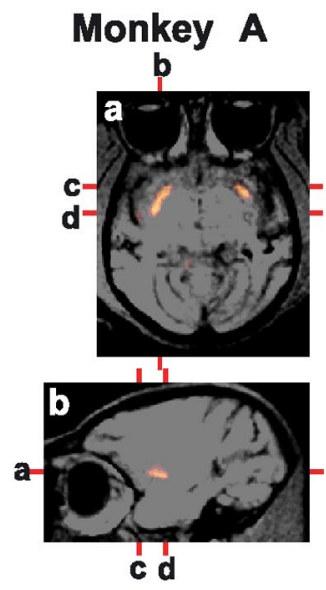

Monkey B

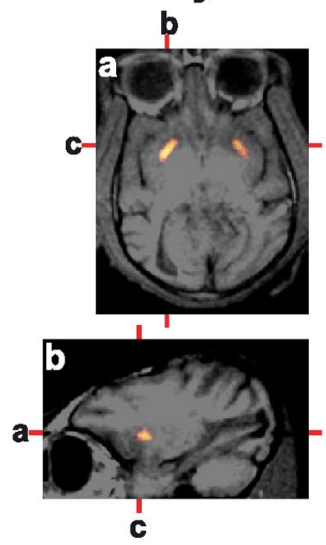

Monkey C
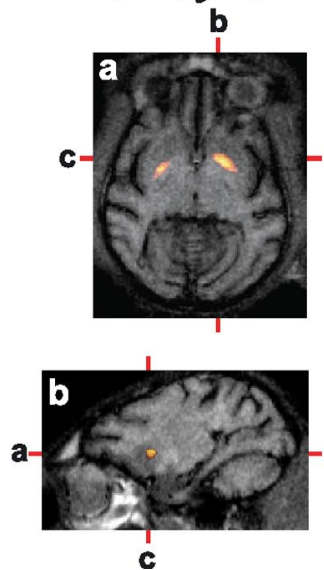

B

Regions with top $20 \%$ activity

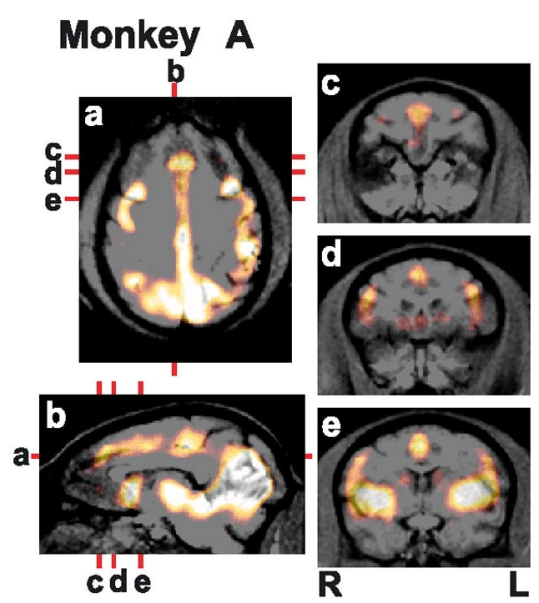

Monkey B

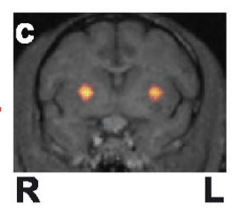

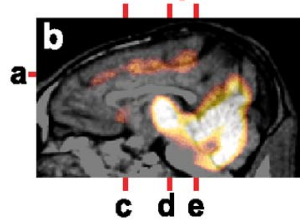

Monkey C

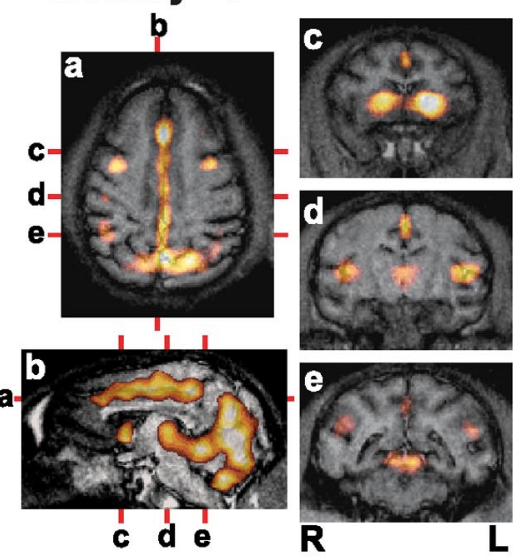

Figure 7. Regions with high levels of rCBF. Regions with rCBF levels in the top $5 \%(\boldsymbol{A})$ and top $20 \%(\boldsymbol{B})$ are illustrated separately for each monkey. Panels in the top left $(\boldsymbol{a})$, bottom left $(\boldsymbol{b})$, and right $(\boldsymbol{c} \boldsymbol{e})$ indicate transverse, sagittal, and coronal brain sections of each monkey, respectively. Other conventions are the same as in Figure 1.

cerned with higher executive control (Miller and Cohen, 2001). It thus appears that resting cognition involves greater executive control than the tasks do. However, it is suggested that the LPFC may show task-induced deactivation when the task is easy but may not show deactivation when the task demands a higher cognitive load (Christoff et al., 2004). All tasks in this study were
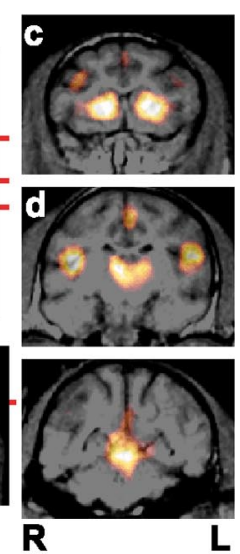

performed by the monkey with almost $100 \%$ accuracy and thus must not be load demanding. When contrasted with the magnitude of activity during the CON task, the magnitude of activity during REST was far larger than that of activity during the WM task (WM-related activity was only slightly higher than CON-related activity) (Kojima et al., 2007, their Figs. 1, 3 ), and thus higher rest-related than WM related activity was observed in the LPFC. Thus, task-induced deactivations observed in the LPFC may be caused, not because resting cognition involved greater executive control, but because the task situation did not demand much executive control.

The OFC is known to be concerned with processing reward information. During the PET scan experiment, the monkeys were allowed to perform the task and obtain the liquid rewards during each of the task conditions but not during REST. It is notable that the OFC and ventral striatum that are activated in relation to reward processing in the human (Porcelli and Delgado, 2009) did not show higher activity during the task performance than during REST in the monkey (supplemental Tables $1 B, 2 B$, available at www.jneurosci.org as supplemental material). The monkeys were fluid restricted and therefore must have been eager to perform the tasks to obtain their reward. Thus, the monkey's desire for the liquid reward might have been very strong during REST. Monkey OFC neurons are related to both reward delivery and reward expectancy (Schultz and Tremblay, 2006). Human neuroimaging studies indicate that the OFC is concerned not only with receiving the reward but also with reward expectancy (O'Doherty et al., 2002). It is speculated that the magnitude of reward expectancy-related activity might have been larger than that of reward delivery-related activity. Conversely, the resting condition may have been frustrating to the monkey that was not allowed to perform the task to obtain the reward. Considering that the OFC is also related to processing aversive stimuli (Thorpe et al., 1983), the resting activity in the OFC may be related to the monkey's frustration associated with the resting condition. Thus, reward expectancy and/or frustration during REST might have induced higher rest-related than taskrelated activity in this brain area.

Neuronal activities concerned with processing appetitive and aversive stimuli have been observed not only in the OFC but also in the LPFC (Thorpe et al., 1983; Hikosaka and Watanabe, 2000; Kobayashi et al., 2006). Human OFC and LPFC are both activated in relation to reward processing (Thut et al., 1997). The rest- 
related activity observed in the regions of the LPFC and OFC in the present study that do not constitute the human default system may also be concerned with internal thought processes, which may involve more motivational and emotional contents.

During REST, we did not attempt to influence or control their thought processes. Thus, it was not surprising that we found individual differences in the rest-related activity in terms of magnitude and regions within the default system (Figs. 1, 3) (supplemental Tables $1 A, 2 A$, available at www.jneurosci.org as supplemental material). Indeed, the number of voxels with task-induced deactivation differed significantly among the three monkeys; for example, the number of voxels with spatial-DR task-induced deactivation was 128,231 , and 591 for monkeys A, B, and C, respectively (supplemental Table $1 A$, available at www.jneurosci.org as supplemental material). In monkeys A and B, single-monkey analysis, but not multi-monkey analysis, revealed significant, nonspatial taskinduced deactivation in the PCC/precuneus, maybe because the two monkeys showed the deactivation in different regions within this brain area.

Although a previous study indicated the highest metabolic activity in the default system in the resting chimpanzee (Rilling et al., 2007), we found the highest rCBF in the dorsal striatum in the resting monkey. Chimpanzees in the previous study remained in the home cage during the $\left[{ }^{18} \mathrm{~F}\right] \mathrm{FDG}$ uptake period. In contrast, the monkeys in our study were sitting in the primate chair in an upright posture. The subtraction between the REST and (any) task condition did not reveal a significant difference in activity in the part of the striatum that showed the highest resting $\mathrm{rCBF}$ in all of the monkeys. Considering also that the monkey moved the right hand from time to time during REST, the high $\mathrm{rCBF}$ in the dorsal striatum during REST in the present study could be caused by both the requirement that the animal sit upright on the primate chair with the head rigidly restrained and the movement of the monkey's right hand.

In all monkeys, there were significant differences between regions showing task-induced deactivation and regions showing the highest level of rCBF during REST. For example, the ventromedial PFC including the medial PFC and OFC showed clear task-induced deactivation, whereas there was no high-level rCBF observed in this brain area during rest in any monkey. Because regions with the highest rCBF may differ depending on the resting condition (such as lying in the bed vs sitting on a chair or liquid restricted vs not restricted), there may also be differences between regions with task-induced deactivation and regions with the highest level of rCBF or metabolic activity on PET or fMRI scans obtained from human in upright position.

Rilling et al. (2007) suggested that the resting state of chimpanzees involves emotionally laden episodic memory retrieval and some level of mental self-projection. Chimpanzees are surely more similar to the human than the monkey with regard to intellectual abilities. The lateral posterior parietal area constitutes the default system in the human and may support internal thought processes by its language-related activity. It is speculated that resting activity observed in this brain area in the chimpanzee, but only weakly observed in the monkey, may be related to much higher cognitive, possibly prelinguistic, operations conducted in this brain area in the chimpanzee than in the monkey. Although some researchers have argued that monkeys may not have a theory of mind (Povinelli et al., 1991), there are data indicating that the monkey has a degree of social intelligence, as exemplified by their capacity for deceptive behavior (Hauser, 1997), altruistic behavior (Burkart et al., 2007), and fairness judgments (Brosnan and De Waal, 2003). Furthermore, a recent study suggested that monkeys demonstrate "theory of mind" abilities when tested in more ecologically relevant situations (Flombaum and Santos, 2005). Considering that monkeys live within a complex social structure and thus are required to process self and others in the context of society, it is not implausible to consider that internal thought processes exist not only in chimpanzees but also in monkeys.

\section{References}

Binder JR, Frost JA, Hammeke TA, Bellgowan PS, Rao SM, Cox RW (1999) Conceptual processing during the conscious resting state: a functional MRI study. J Cogn Neurosci 11:80-95.

Brosnan SF, De Waal FB (2003) Monkeys reject unequal pay. Nature 425:297-299.

Burkart JM, Fehr E, Efferson C, van Schaik CP (2007) Other-regarding preferences in a non-human primate: common marmosets provision food altruistically. Proc Natl Acad Sci U S A 104:19762-19766.

Christoff K, Ream JM, Gabrieli JD (2004) Neural basis of spontaneous thought processes. Cortex 40:623-630.

Fair DA, Cohen AL, Dosenbach NU, Church JA, Miezin FM, Barch DM, Raichle ME, Petersen SE, Schlaggar BL (2008) The maturing architecture of the brain's default network. Proc Natl Acad Sci USA 105:4028-4032.

Flombaum JI, Santos LR (2005) Rhesus monkeys attribute perceptions to others. Curr Biol 15:447-452.

Fox MD, Snyder AZ, Vincent JL, Corbetta M, Van Essen DC, Raichle ME (2005) The human brain is intrinsically organized into dynamic, anticorrelated functional networks. Proc Natl Acad Sci U S A 102:9673-9678.

Fransson P (2005) Spontaneous low-frequency BOLD signal fluctuations: an $\mathrm{fMRI}$ investigation of the resting-state default mode of brain function hypothesis. Hum Brain Mapp 26:15-29.

Greicius MD, Flores BH, Menon V, Glover GH, Solvason HB, Kenna H, Reiss AL, Schatzberg AF (2007) Resting-state functional connectivity in major depression: abnormally increased contributions from subgenual cingulate cortex and thalamus. Biol Psychiatry 62:429-437.

Gusnard DA, Raichle ME, Raichle ME (2001) Searching for a baseline: functional imaging and the resting human brain. Nat Rev Neurosci 2:685-694.

Hauser MD (1997) Minding the behaviour of deception. In: Machiavellian intelligence II: extensions and evaluations (Whiten A, Byrne RW, eds), pp 112-145. Cambridge, UK: Cambridge UP.

Hayden BY, Nair AC, McCoy AN, Platt ML (2008) Posterior cingulate cortex mediates outcome-contingent allocation of behavior. Neuron 60:19-25.

Hayden BY, Smith DV, Platt ML (2009) Electrophysiological correlates of default-mode processing in macaque posterior cingulate cortex. Proc Natl Acad Sci U S A 106:5948-5953.

Hikosaka K, Watanabe M (2000) Delay activity of orbital and lateral prefrontal neurons of the monkey varying with different rewards. Cereb Cortex 10:263-271.

Kelley WM, Macrae CN, Wyland CL, Caglar S, Inati S, Heatherton TF (2002) Finding the self? An event-related fMRI study. J Cogn Neurosci 14:785-794.

Kennedy DP, Redcay E, Courchesne E (2006) Failing to deactivate: resting functional abnormalities in autism. Proc Natl Acad Sci USA 103:8275-8280.

Kobayashi S, Nomoto K, Watanabe M, Hikosaka O, Schultz W, Sakagami M (2006) Influences of rewarding and aversive outcomes on activity in macaque lateral prefrontal cortex. Neuron 51:861-870.

Kojima T, Onoe H, Hikosaka K, Tsutsui K, Tsukada H, Watanabe M (2007) Domain-related differentiation of working memory in the Japanese macaque (Macaca fuscata) frontal cortex: a positron emission tomography study. Eur J Neurosci 25:2523-2535.

Liang M, Zhou Y, Jiang T, Liu Z, Tian L, Liu H, Hao Y (2006) Widespread functional disconnectivity in schizophrenia with resting-state functional magnetic resonance imaging. Neuroreport 17:209-213.

Lustig C, Snyder AZ, Bhakta M, O’Brien KC, McAvoy M, Raichle ME, Morris JC, Buckner RL (2003) Functional deactivations: change with age and dementia of the Alzheimer type. Proc Natl Acad Sci USA 100:14504-14509.

Mason MF, Norton MI, Van Horn JD, Wegner DM, Grafton ST, Macrae CN 
(2007) Wandering minds: the default network and stimulus independent thought. Science 315:393-395.

Mazoyer B, Zago L, Mellet E, Bricogne S, Etard O, Houdé O, Crivello F, Joliot M, Petit L, Tzourio-Mazoyer N (2001) Cortical networks for working memory and executive functions sustain the conscious resting state in man. Brain Res Bull 54:287-298.

McKiernan KA, Kaufman JN, Kucera-Thompson J, Binder JR (2003) A parametric manipulation of factors affecting task-induced deactivation in functional neuroimaging. J Cogn Neurosci 15:394-408.

Miller EK, Cohen JD (2001) An integrative theory of prefrontal cortex function. Annu Rev Neurosci 24:167-202.

Minoshima S, Giordani B, Berent S, Frey KA, Foster NL, Kuhl DE (1997) Metabolic reduction in the posterior cingulate cortex in very early Alzheimer's disease. Ann Neurol 42:85-94.

O’Doherty JP, Deichmann R, Critchley HD, Dolan RJ (2002) Neural responses during anticipation of a primary taste reward. Neuron 33: $815-826$.

Onoe H, Komori M, Onoe K, Takechi H, Tsukada H, Watanabe Y (2001) Cortical networks recruited for time perception, a monkey positron emission tomography (PET) study. Neuroimage 13:37-45.

Paxinos G, Huang X-F, Toga AW (2000) The rhesus monkey brain in stereotaxic coordinates. San Diego: Academic.

Porcelli AJ, Delgado MR (2009) Reward processing in the human brain: insights from fMRI. In: Handbook of reward and decision making (Dreher JC, Tremblay L, eds), pp 159-178. Oxford: Academic.

Povinelli DJ, Parks KA, Novak MA (1991) Do rhesus monkeys (Macaca mulatta) attribute knowledge and ignorance to others? J Comp Psychol 105:318-325.

Raichle ME, MacLeod AM, Snyder AZ, Powers WJ, Gusnard DA, Shulman
GL (2001) A default mode of brain function. Proc Natl Acad Sci U S A 98:676-682.

Rilling JK, Barks SK, Parr LA, Preuss TM, Faber TL, Pagnoni G, Bremner JD, Votaw JR (2007) A comparison of resting-state brain activity in humans and chimpanzees. Proc Natl Acad Sci U S A 104:17146-17151.

Rushworth MF, Buckley MJ, Behrens TE, Walton ME, Bannerman DM (2007) Functional organization of the medial frontal cortex. Curr Opin Neurobiol 17:220-227.

Saleem KS, Logothetis NK (2007) A combined MRI and histology atlas of the rhesus monkey brain in stereotaxic coordinates. London: Academic.

Schultz W, Tremblay L (2006) Involvement of primate orbitofrontal neurons in reward, uncertainty, and learning. In: The orbitofrontal cortex (Zald DH, Rauch SL, eds), pp 173-198. Oxford: Oxford UP.

Shulman GL, Fiez J, Corbetta M, Buckner R, Miezin FM, Raichle ME, Petersen S (1997) Common blood flow changes across visual task. II. Decreases in cerebral cortex. J Cogn Neurosci 9:648-663.

Thorpe SJ, Rolls ET, Maddison S (1983) The orbitofrontal cortex: neuronal activity in the behaving monkey. Exp Brain Res 49:93-115.

Thut G, Schultz W, Roelcke U, Nienhusmeier M, Missimer J, Maguire RP, Leenders KL (1997) Activation of the human brain by monetary reward. Neuroreport 8:1225-1228.

Tian L, Jiang T, Wang Y, Zang Y, He Y, Liang M, Sui M, Cao Q, Hu S, Peng M, Zhuo Y (2006) Altered resting-state functional connectivity patterns of anterior cingulate cortex in adolescents with attention deficit hyperactivity disorder. Neurosci Lett 400:39-43.

Vincent JL, Patel GH, Fox MD, Snyder AZ, Baker JT, Van Essen DC, Zempel JM, Snyder LH, Corbetta M, Raichle ME (2007) Intrinsic functional architecture in the anaesthetized monkey brain. Nature 447:83-86. 